\title{
Should a Sentinel Node Biopsy Be Performed in Patients with High-Risk Breast Cancer?
}

\section{Citation}

Westover, Kenneth D., M. Brandon Westover, Eric P. Winer, Andrea L. Richardson, J. Dirk Iglehart, and Rinaa S. Punglia. 2011. Should a sentinel node biopsy be performed in patients with high-risk breast cancer?. International Journal of Breast Cancer 973245.

\section{Published Version}

doi:10.4061/2011/973245

\section{Permanent link}

http://nrs.harvard.edu/urn-3:HUL.InstRepos:8704123

\section{Terms of Use}

This article was downloaded from Harvard University's DASH repository, and is made available under the terms and conditions applicable to Other Posted Material, as set forth at http:// nrs.harvard.edu/urn-3:HUL.InstRepos:dash.current.terms-of-use\#LAA

\section{Share Your Story}

The Harvard community has made this article openly available.

Please share how this access benefits you. Submit a story.

\section{Accessibility}




\title{
Should a Sentinel Node Biopsy Be Performed in Patients with High-Risk Breast Cancer?
}

\author{
Kenneth D. Westover, ${ }^{1}$ M. Brandon Westover, ${ }^{2}$ Eric P. Winer, ${ }^{3}$ Andrea L. Richardson, ${ }^{4}$ \\ J. Dirk Iglehart, ${ }^{5}$ and Rinaa S. Punglia ${ }^{6}$
}

${ }^{1}$ Harvard Radiation Oncology Program, Boston, MA 02115, USA

${ }^{2}$ Department of Neurology, Dana-Farber Cancer Institute, Brigham and Women's Hospital and Harvard Medical School, Boston, MA 02115, USA

${ }^{3}$ Department of Medical Oncology, Dana-Farber Cancer Institute, Brigham and Women's Hospital and Harvard Medical School, Boston, MA 02115, USA

${ }^{4}$ Department of Pathology, Dana-Farber Cancer Institute, Brigham and Women's Hospital and Harvard Medical School, Boston, MA 02115, USA

${ }^{5}$ Department of Surgery, Dana-Farber Cancer Institute, Brigham and Women's Hospital and Harvard Medical School, Boston, MA 02115, USA

${ }^{6}$ Department of Radiation Oncology, Dana-Farber Cancer Institute, Brigham and Women's Hospital and Harvard Medical School, Boston, MA 02115, USA

Correspondence should be addressed to Rinaa S. Punglia, rpunglia@partners.org

Received 13 April 2011; Revised 14 June 2011; Accepted 23 June 2011

Academic Editor: Luciane R. Cavalli

Copyright (๑) 2011 Kenneth D. Westover et al. This is an open access article distributed under the Creative Commons Attribution License, which permits unrestricted use, distribution, and reproduction in any medium, provided the original work is properly cited.

\begin{abstract}
A negative sentinel lymph node (SLN) biopsy spares many breast cancer patients the complications associated with lymph node irradiation or additional surgery. However, patients at high risk for nodal involvement based on clinical characteristics may remain at unacceptably high risk of axillary disease even after a negative SLN biopsy result. A Bayesian nomogram was designed to combine the probability of axillary disease prior to nodal biopsy with customized test characteristics for an SLN biopsy and provides the probability of axillary disease despite a negative SLN biopsy. Users may individualize the sensitivity of an SLN biopsy based on factors known to modify the sensitivity of the procedure. This tool may be useful in identifying patients who should have expanded upfront exploration of the axilla or comprehensive axillary irradiation.
\end{abstract}

\section{Introduction}

In breast cancer, metastases to the axilla are associated with an increased risk of distant micrometastatic disease [1-3]. Sentinel lymph node (SLN) biopsy has become standard practice for evaluating the axilla in patients without palpable lymph nodes [4]. This procedure involves injection of a tracer, usually a radioactive colloid, alone or in combination with dye, into the tissue surrounding a tumor. Lymph nodes with evidence of uptake are surgically removed. The SLN procedure typically yields $1-5$ nodes for pathologic examination whereas full axillary lymph node dissection (ALND) can yield greater than 20 nodes when taken to completion.
On the other hand, SLN biopsy is associated with less pain, lower rates of postsurgical lymphedema, and better arm mobility when compared to full ALND [4]. Many patients with a positive SLN biopsy go on to have ALND for both diagnostic and therapeutic purposes. However, there is growing evidence that axillary irradiation may be used instead of ALND in select cases with excellent results [5]. Indeed a recent randomized trial showed that ALND offered no benefit over SNL biopsy in terms of local control or survival to women with early clinical stage breast cancer who also received radiation therapy [6].

Like any diagnostic test, SLN biopsy can yield falsenegative results. Several factors can affect the sensitivity of 
TABLE 1: Factors influencing the sensitivity of SLN biopsy.

\begin{tabular}{lcc}
\hline Factor & Sensitivity & Reference \\
\hline T1 & $89.7-93.3 \%$ & {$[4,7,8]$} \\
T2-T3 & $82.0-92.6 \%$ & {$[7,9]$} \\
Grade & & {$[10]$} \\
1 & $95.7 \%$ & \\
3 & $90.4 \%$ & \\
Skill of surgeon & $72.4-100 \%$ & {$[8]$} \\
Method & & {$[11]$} \\
$\quad$ Combined dye and isotope & $86.3-96.0 \%$ & \\
$\quad$ Dye & $85.7-90.4 \%$ & \\
$\quad$ Isotope & $86.3-97.8 \%$ & \\
Number of SLN removed & & {$[10,12]$} \\
1 & $82.3-89.1 \%$ & \\
3 & $93.1-98.9 \%$ & \\
5 & $99 \%$ & {$[8]$} \\
Medial tumor & Decreases & \\
Age $>50$ & Decreases & {$[10,13]$} \\
Obesity & Decreases &
\end{tabular}

an axillary SLN biopsy (Table 1). Large tumors have been associated with decreased sensitivity (equivalently, higher false-negative rates), perhaps because they access a greater number of local lymphatic pathways and therefore have the potential for spreading to a larger distribution of nodes [7,9]. Age-related fatty changes in nodes may decrease the capacity for dye or isotope uptake [8]. Medially located tumors may drain more frequently to internal mammary nodes than tumors located centrally or in the lateral breast [8]. Finally, two prospective studies suggest that the sensitivity of SLN biopsy correlates with the number of nodes removed [10, 12]. It should be noted that as techniques and protocols have improved, the sensitivity of SLN biopsy has generally improved. Nevertheless, the procedure remains imperfect, and most recent studies demonstrate false-negative rates in the range of $5-10 \%$ for small tumors $[10,12]$.

Breast cancer risk calculators are being increasingly used to guide adjuvant systemic and local treatment [14-17]. Using such a calculator, the probability of axillary nodal involvement for a given patient can be estimated prior to SLN biopsy based on a number of prognostic factors including age, tumor size, and histopathological features of the breast cancer. One example of such a risk calculator was developed at Memorial Sloan Kettering (MSKCC) and is available for use online. This calculator was originally intended to spare low-risk patients an SLN biopsy when the probability of nodal involvement is low [14]. However, this calculated probability may also benefit high-risk patients when used in combination with estimates of the false-negative rate of SLN biopsy to calculate the risk of having residual nodal disease in the setting of a negative SLN. We developed a nomogram that combines this probability of axillary disease with estimates of the sensitivity of SLN biopsy, to calculate the risk of residual axillary disease despite a negative SLN biopsy.

\section{Methods}

Bayes' rule combines the pretest probability of a given diagnosis with results from a test with known sensitivity and specificity to yield a posttest probability of having the diagnosis. In this analysis, the pretest probability is the probability of having axillary disease prior to any nodal evaluation; the posttest probability is the probability of axillary disease given a negative SLN biopsy; the false-negative rate (1-sensitivity) of SLN biopsy can be estimated from Table 1 . The specificity of SLN biopsy is by definition equal to one (equivalently, the probability of a positive SLN in the absence of lymph node involvement disease is zero).

In this setting, Bayes' rule takes the following form:

$$
\text { Post }=\text { pre } * \frac{(1-\text { sens })}{\text { pre } *(1-\text { sens })+(1-\text { pre })(\text { spec })} \text {. }
$$

With "post" and "pre" defined as posttest and pretest probabilities, respectively, "sens" defined as the sensitivity of SLN biopsy, and "spec" defined as the specificity of the procedure, which in our situation is 1 .

Using this formula we can estimate the probability that a breast cancer patient has residual axillary disease despite a negative SLN biopsy. A Bayesian nomogram was constructed in MATLAB (MathWorks, v7.8) using the mathematical relationship above. We used a range of pretest probabilities from 5 to $85 \%$ and a 4 estimates of sensitivity $(80 \%, 85 \%$, $90 \%$, and $95 \%$ ) for the SLN biopsy procedure.

\section{Results}

We created a Bayesian nomogram for the probability of axillary nodal involvement despite a negative SLN biopsy (Figure 1). The nomogram was designed to be flexible in order to accommodate a variety of clinical scenarios. A range of sensitivity values are displayed along the middle axis as discrete points; the appropriate value for a given patient can be estimated using Table 1. A line drawn through a given pretest probability and sensitivity point will intersect with the appropriate posttest probability (probability of having residual axillary disease despite a negative SLN biopsy) on the right-hand axis.

For example, the nomogram can be used to calculate the risk of residual nodal disease in a 62-year-old woman who presents after a lumpectomy revealing a $1.5 \mathrm{~cm}$, grade 2 invasive ductal carcinoma, hormone receptor-negative, with no lymphovascular invasion and an SLN biopsy yielding 3 negative nodes. According to the MSKCC model her risk of axillary disease prior to SLN biopsy is 19\%. Assuming 95\% for the sensitivity of SLN biopsy in this situation, the nomogram reveals that the probability of having residual axillary disease is $1.2 \%$. Even if the sensitivity of SLN biopsy was assumed to be $85 \%$, the posttest probability remains low at $3.4 \%$.

Likewise, the nomogram can be used to calculate the probability of residual axillary disease despite a negative SLN biopsy in a woman at higher risk of axillary involvement. A 64 -year-old patient with a $2 \mathrm{~cm}$, grade 3 , hormone receptor-positive, invasive ductal carcinoma with lymphovascular 


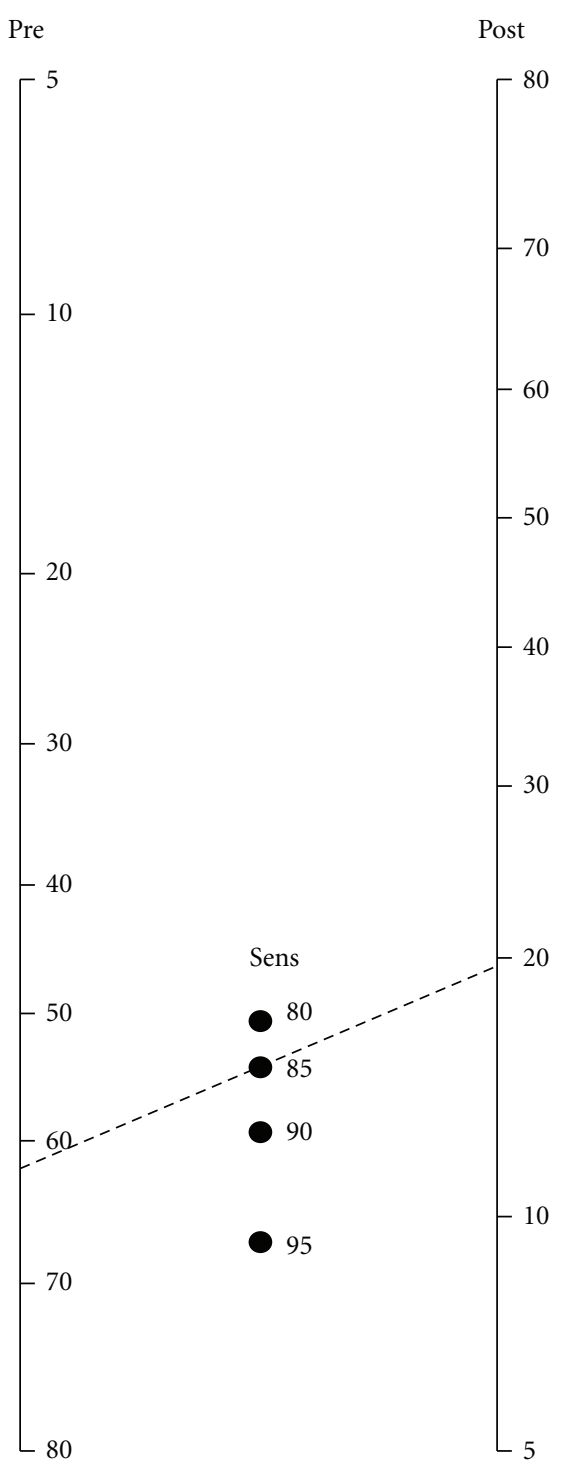

FiguRE 1: Bayesian nomogram for probability of metastatic disease as a function of pretest probability and negative SLN biopsy. The "pre" or pretest probability of axillary disease can be estimated using a risk calculator such as the one described [14]. The "sens" or sensitivity of SLN biopsy can be estimated using Table 1. Positions for the central dots are calculated assuming a SLN biopsy sensitivity of $80 \%, 85 \%, 90 \%$, or $95 \%$; specificity is assumed to be $100 \%$. The calculation for the example patient is shown by the dotted line: if we assume sensitivity of $85 \%$ for SLN biopsy and a pretest probability of $62 \%$, the posttest probability for axillary disease is $20 \%$ for this patient even in the presence of a negative SLN biopsy.

invasion has a $62 \%$ pretest probability of metastases to the axilla based on her pathology. Using a sensitivity of $85 \%$ for SLN biopsy, the nomogram reveals that the probability of residual axillary nodal involvement is $20 \%$. In other words, despite a negative SLN biopsy, she still has a $20 \%$ probability of finding metastatic disease with completion ALND. If the SLN biopsy procedure has a $95 \%$ sensitivity the nomogram reveals that the risk of additional axillary disease after a negative SLN biopsy decreases to $7.5 \%$.
TABLE 2: Calculated posttest probability of residual axillary disease despite negative SLN biopsy for a range of pretest probabilities for axillary disease with varying sensitivities of SLN biopsy.

\begin{tabular}{lccc}
\hline & \multicolumn{3}{c}{ Posttest probability of axillary disease } \\
Pretest & Sens 0.85 & Sens 0.90 & Sens 0.95 \\
\hline 0.05 & 0.008 & 0.005 & 0.003 \\
0.1 & 0.016 & 0.011 & 0.006 \\
0.2 & 0.036 & 0.024 & 0.012 \\
0.3 & 0.060 & 0.041 & 0.021 \\
0.4 & 0.091 & 0.063 & 0.032 \\
0.5 & 0.130 & 0.091 & 0.048 \\
0.6 & 0.184 & 0.130 & 0.070 \\
0.7 & 0.259 & 0.189 & 0.104 \\
0.8 & 0.375 & 0.286 & 0.167 \\
0.9 & 0.574 & 0.474 & 0.310 \\
\hline
\end{tabular}

Table 2 provides a summary of findings for the posttest probability of residual axillary disease despite negative SLN biopsy for a range of pretest probabilities of axillary disease prior to SLN biopsy at each of 3 different sensitivities for the SLN procedure.

\section{Discussion}

The presence of axillary disease is the most important prognostic factor in breast cancer. Disease in the axilla can indicate biological aggressiveness and extent of tumor involvement, often suggesting systemic spread and the need for additional therapy. In addition, the link between locoregional disease control and overall survival in breast cancer has been firmly established by meta-analyses of randomized data [18]. The potential importance of ALND in select patients is underscored by a recent analysis which suggested a survival benefit for women with macroscopic nodal disease that received ALND as compared to women with SLN biopsy alone [19]. Additionally the NCIC CTG MA.20 trial which randomized patients with high-risk breast cancer to postoperative wholebreast (WB) radiotherapy alone versus WB plus regional nodal irradiation showed improved locoregional control and an even greater improvement in distant disease control in the arm with regional nodal irradiation [20]. Therefore, the risks of more extensive axillary treatment versus the risks of missing occult disease must be carefully considered.

Our nomogram is intended to be flexible and enable increased personalization of cancer care. Specifically, our analysis is most applicable to two clinical scenarios and argues that

(1) for a patient who had a negative SLN biopsy, but still has a high posttest probability of axillary disease, comprehensive axillary radiation may be warranted;

(2) for a patient who has not yet undergone any axillary surgery, who has a high pretest probability of positive axillary nodes based on clinical features and who also has clinical characteristics that might decrease the 
sensitivity of SLN biopsy (as in Table 1), expanded axillary assessment up front may be warranted.

These conclusions seek to limit overtreatment in the form of multiple surgeries (SLN biopsy followed by ALND) and undertreatment in the form of omission of axillary radiation in breast patients with high-risk disease.

We created a nomogram to estimate the risk of residual axillary disease despite a negative SLN biopsy as a function of the sensitivity of the SLN biopsy procedure and the pretest probability of axillary disease prior to axillary evaluation. Our nomogram reveals that for patients with a high pretest probability of axillary metastases and factors associated with a lower SLN biopsy sensitivity, the posttest probability of axillary disease often remains high despite a negative biopsy. While SLN biopsy is the appropriate test for most breast cancer patients, a preemptive expanded assessment of the axilla may be a better choice for high-risk patients or in cases where an SLN biopsy is predicted to be less sensitive (Table 1).

In contrast to other probability calculators which estimate the risk of nonsentinel axillary nodal disease in a woman with breast cancer only after a positive SLN, our decision tool estimates the probability of nonsentinel axillary nodal disease without prior pathologic assessment of the axilla [15$17,21]$. The posttest probability obtained from the nomogram presented can then be assessed to be acceptable or not based on the specific clinical scenario. For example, a predicted posttest probability greater than $20-25 \%$ may warrant consideration of a more thorough axillary assessment upfront. Similarly, in cases where a negative SLN biopsy has already been obtained, a posttest probability of greater than $10 \%$ may suggest the need for the addition of axillary radiation.

In patients identified by the nomogram to have an unacceptably high-risk of residual axillary disease despite negative SLN biopsy, more aggressive preemptive exploration of the axilla will necessarily mean a higher risk of lymphedema, nerve injury and general surgical complications compared to SLN biopsy; nevertheless, with modern ALND where only levels I-II are removed, these risks are less than observed historically [20]. Another option may be a less morbid lymph node sampling procedure as was reported in the UK, where at least 4 palpable lymph nodes are obtained by dissection starting at the axillary tail [22]. For patients where a negative SLN biopsy has already been obtained, but a high posttest probability of axillary disease remains, the addition of axillary radiation therapy could be considered in lieu of completion axillary dissection.

Use of this nomogram after a breast biopsy is not expected to result in excessive axillary treatment because it is likely to slightly underestimate rather than overestimate the probability of residual nodal disease. This is because the MSKCC risk calculator used above to obtain a pretest probability of having nodal disease was validated with information from complete pathologic specimens whereas, in practice, physicians are likely to substitute incomplete biopsy specimens such as findings from core needle biopsy, resulting in underdetection (due to undersampling) of certain negative prognostic factors, such as lymphovascular invasion, multifocality, and higher-grade tumor areas, which if found, increase the probability of having nodal disease. Therefore, use of only biopsy information would lead to underestimation of the pretest probability of having axillary involvement, which in turn would lead to underestimation of the posttest probability when using the nomogram. Another possible source of error associated with the use of incomplete biopsy specimens is that tumor size estimate entered into the MSKCC risk calculator must be estimated indirectly, based on imaging. However, assessment of tumor size by MRI, mammography and ultrasound does appear to correlate well with size as determined by pathologic exam $[23,24]$.

Although the recently published ACOSOG Z0011 and NSABP B-32 trials show similar rates of local control in both their SLN alone and ALND arms, the patients included in these trials were conservatively chosen and by definition had a low-risk profile $[6,25]$. For the average patient in these trials, the risk of additional nodal disease after a negative SLN biopsy according to our nomogram would be under $6 \%$ even when using a low value of $85 \%$ as the sensitivity for SLN biopsy. Therefore our nomogram would not change the management of the average patient on these trials.

For high-risk patients systemic therapy recommendations are also unlikely to change based on the output of this nomogram, because many of the factors which prompt addition of adjuvant therapy are the same factors that increase the pretest (and therefore also posttest) probability of having axillary disease. However, with regard to local management of the axilla, using this nomogram may change management, especially in light of the NCIC CTG MA.20 trial which included high-risk patients and suggests that residual untreated nodal disease may affect distant disease even in the absence of a clinically evident nodal recurrence.

This nomogram is anticipated to apply to the small proportion of breast cancer patients who present with clinically high-risk disease. Randomized studies for this subset are difficult to perform because most breast cancer patients present with early-stage disease. Therefore, Bayesian estimation of risk based on a mathematically sound extrapolation from available data is especially useful in this clinical situation. Our nomogram is a particularly important tool for this group of high-risk patients because they may benefit from more extensive surgery or axillary radiation.

\section{Acknowledgments}

This paper was funded by the National Institutes of Health (Grant no. 1K07 CA118269 to R. S. Punglia).

\section{References}

[1] J. J. Albertini, G. H. Lyman, C. Cox et al., "Lymphatic mapping and sentinel node biopsy in the patient with breast cancer," Journal of the American Medical Association, vol. 276, no. 22, pp. 1818-1822, 1996.

[2] S. Zurrida, A. Morabito, V. Galimberti et al., "Importance of the level of axillary involvement in relation to traditional 
variables in the prognosis of breast cancer," International Journal of Oncology, vol. 15, no. 3, pp. 475-480, 1999.

[3] J. W. Berg, "The significance of axillary node levels in the study of breast carcinoma," Cancer, vol. 8, no. 4, pp. 776-778, 1955.

[4] U. Veronesi, G. Paganelli, G. Viale et al., "A randomized comparison of sentinel-node biopsy with routine axillary dissection in breast cancer," New England Journal of Medicine, vol. 349, no. 6, pp. 546-553, 2003.

[5] "Impact of omission of completion axillary lymph node dissection (cALND) or axillary radiotherapy (ax RT) in breast cancer patients with micrometastases $(\mathrm{pN} 1 \mathrm{mi})$ or isolated tumor cells ( $\mathrm{pN} 0[\mathrm{i}+])$ in the sentinel lymph node $(\mathrm{SN})$ : results from the MIRROR study-Tjan-Heijnen et al," CRA506ASCO Meeting Abstracts, vol. 27, no. 15, 2009.

[6] A. E. Giuliano, L. McCall, P. Beitsch et al., "Locoregional recurrence after sentinel lymph node dissection with or without axillary dissection in patients with sentinel lymph node metastases," Annals of Surgery, vol. 252, no. 3, pp. 426-433, 2010.

[7] U. Veronesi, G. Paganelli, V. Galimberti et al., "Sentinel-node biopsy to avoid axillary dissection in breast cancer with clinically negative lymph-nodes," Lancet, vol. 349, no. 9069, pp. 1864-1867, 1996.

[8] D. Krag, D. Weaver, T. Ashikaga et al., "The Sentinel node in breast cancer. A multicenter validation study," New England Journal of Medicine, vol. 339, no. 14, pp. 941-946, 1998.

[9] B. J. O'Hea, A. D. Hill, A. M. El-Shirbiny et al., "Sentinel lymph node-biopsy in breast cancer: initial experience at memorial sloan-kettering cancer center," Journal of the American College of Surgeons, vol. 186, no. 4, pp. 423-427, 1998.

[10] A. Goyal, R. G. Newcombe, A. Chhabra, and R. E. Mansel, "Factors affecting failed localisation and false-negative rates of sentinel node biopsy in breast cancer-results of the ALMANAC validation phase," Breast Cancer Research and Treatment, vol. 99, no. 2, pp. 203-208, 2006.

[11] J. M. Harris, Diseases of the Breast, Lippicott Williams \& Wilkins, Philadelphia, Pa, USA, 2000.

[12] D. N. Krag, S. J. Anderson, T. B. Julian et al., "Technical outcomes of sentinel-lymph-node resection and conventional axillary-lymph-node dissection in patients with clinically node-negative breast cancer: results from the NSABP B-32 randomised phase III trial," Lancet Oncology, vol. 8, no. 10, pp. 881-888, 2007.

[13] A. M. Derossis, J. V. Fey, H. S. Cody Jr., and P. I. Borgen, "Obesity influences outcome of sentinel lymph node biopsy in early-stage breast cancer," Journal of the American College of Surgeons, vol. 197, no. 6, pp. 896-901, 2003.

[14] J. L. Bevilacqua, M. W. Kattan, J. V. Fey et al., "Doctor, what are my chances of having a positive sentinel node? A validated nomogram for risk estimation," Journal of Clinical Oncology, vol. 25, no. 24, pp. 3670-3679, 2007.

[15] K. J. V. Zee, D. M. E. Manasseh, J. L. Bevilacqua et al., "A nomogram for predicting the likelihood of additional nodal metastases in breast cancer patients with a positive sentinel node biopsy," Annals of Surgical Oncology, vol. 10, no. 10, pp. 1140-1151, 2003.

[16] A. Pal, E. Provenzano, S. W. Duffy, S. E. Pinder, and A. D. Pumshotham, "A model for predicting non-sentinel lymph node metastatic disease when the sentinel lymph node is positive," British Journal of Surgery, vol. 95, no. 3, pp. 302-309, 2008.

[17] H. E. Kohrt, R. A. Olshen, H. R. Bermas et al., "New models and online calculator for predicting non-sentinel lymph node status in sentinel lymph node positive breast cancer patients," BMC Cancer, vol. 8, article 66, 2008.
[18] M. Clarke, R. Collins, S. Darby et al., "Effects of radiotherapy and of differences in the extent of surgery for early breast cancer on local recurrence and 15-year survival: an overview of the randomised trials," Lancet, vol. 366, no. 9503, pp. 20872106, 2005.

[19] K. Y. Bilimoria, D. J. Bentrem, N. M. Hansen et al., "Comparison of sentinel lymph node biopsy alone and completion axillary lymph node dissection for node-positive breast cancer," Journal of Clinical Oncology, vol. 27, no. 18, pp. 29462953, 2009.

[20] T. Tominaga, S. Takashima, and M. Danno, "Randomized clinical trial comparing level II and level III axillary node dissection in addition to mastectomy for breast cancer," British Journal of Surgery, vol. 91, no. 1, pp. 38-43, 2004.

[21] E. Barranger, C. Coutant, A. Flahault, Y. Delpech, E. Darai, and S. Uzan, "An axilla scoring system to predict nonsentinel lymph node status in breast cancer patients with sentinel lymph node involvement," Breast Cancer Research and Treatment, vol. 91, no. 2, pp. 113-119, 2005.

[22] U. Chetty, W. Jack, R. J. Prescott, C. Tyler, and A. Rodger, "Management of the axilla in operable breast cancer treated by breast conservation: a randomized clinical trial," British Journal of Surgery, vol. 87, no. 2, pp. 163-169, 2000.

[23] J. Kepple, R. Layeeque, V. S. Klimberg et al., "Correlation of magnetic resonance imaging and pathologic size of infiltrating lobular carcinoma of the breast," American Journal of Surgery, vol. 190, no. 4, pp. 623-627, 2005.

[24] F. Tresserra, J. Feu, P. J. Grases, B. Navarro, X. Alegret, and A. Férnandez-Cid, "Assessment of breast cancer size: sonographic and pathologic correlation," Journal of Clinical Ultrasound, vol. 27, no. 9, pp. 485-491, 1999.

[25] D. N. Krag, S. J. Anderson, T. B. Julian et al., "Sentinellymph-node resection compared with conventional axillarylymph-node dissection in clinically node-negative patients with breast cancer: overall survival findings from the NSABP B-32 randomised phase 3 trial," The Lancet Oncology, vol. 11, no. 10, pp. 927-933, 2010. 